\title{
Sensitivity analysis of transonic flow past a NASA airfoil/wing with spoiler deployments
}

\author{
Alexander Kuzmin* \\ St. Petersburg State University, St. Petersburg, Russia
}

\begin{abstract}
Transonic flow past a NASA SC(2)-0710 airfoil with deployments of a spoiler up to $6^{\circ}$ was studied numerically. We consider angles of attack from $-0.6^{\circ}$ to $0.6^{\circ}$ and free-stream Mach numbers from 0.81 to 0.86 . Solutions of the unsteady Reynoldsaveraged Navier-Stokes equations were obtained with a finite-volume solver using several turbulence models. Both stationary and time-dependent deployments of the spoiler were examined. The study revealed the existence of narrow bands of the Mach number, angle of attack, and spoiler deflection angle, in which the flow was extremely sensitive to small perturbations. Simulations of 3D flow past a swept wing confirmed the flow sensitivity to small perturbations of boundary conditions.
\end{abstract}

Key words: airfoil, spoiler, local supersonic regions, interaction, adverse conditions, aerodynamic coefficients

\section{Introduction}

Since the 2000s, a development of numerical methods has made it possible to accurately simulate transonic flow over flaps and spoilers with fixed deflection angles [1-3]. Also, several studies addressed unsteady flow over time-dependent control surfaces [4-6]. However, the flow stability and sensitivity to perturbations under transonic conditions were not thoroughly investigated.

Deflections of a spoiler modify the airfoil/wing geometry in such a way that the upper surface becomes nearly flat or concave in a vicinity of the spoiler hinge. In the 2000s, numerical studies demonstrated a high sensitivity of transonic flow over airfoils comprising a flat or nearly flat arc to small perturbations. This sensitivity was caused by an interaction between two supersonic regions that arise and expand on the arc as the free-stream Mach number increases. The expansion followed by a coalescence of the supersonic regions changes pressure distributions crucially on the airfoil and switches the flow to a regime with a single supersonic region. Both flow regimes (with double and single supersonic regions) can be steady or exhibit slight oscillations due to instability of the separated boundary layer (i.e., a buffet onset, near the trailing edge). This phenomenon was scrutinized for a number of symmetric profiles and an asymmetric J-78 airfoil, the upper surface of which is nearly flat in the midchord region [7]. Also, the flow sensitivity to small perturbations was investigated for a Whitcomb airfoil with upward deflections of an aileron [8].

In this paper, we use the concept of the interaction of closely spaced supersonic regions, to analyze transonic flow past a NASA SC(2)-0710 airfoil/wing with a flap-type spoiler. Emphasis is placed on the flow physics and determination of free-stream conditions that allow jumps in aerodynamic coefficients.

\section{Formulation of the problem}

In Sections 4-7, we consider 2D turbulent flow past a 10\%-thick supercritical NASA SC(2)-0710 airfoil [9]:

$$
y=y_{0710}(x) \text { at } 0<x<1
$$

where $x$ and $y$ are non-dimensional Cartesian coordinates.
This is an Open Access article distributed under the terms of the Creative Commons Attribution Non-Commercial License (http://creativecommons.org/licenses/by$\mathrm{nc} / 3.0 /$ which permits unrestricted non-commercial use, distribution, and reproduction in any medium, provided the original work is properly cited. (c) * Dr.Sc., Head Researcher, Corresponding author : alexander.kuzmin@pobox. spbu.ru 
To simulate spoiler deployment, we introduce a modification of the airfoil's upper surface in the interval $0.55 \leq x \leq$ 0.77 as follows:

the outer surface of the spoiler :

$$
y=y_{0710}(x)+(x-0.55) \tan \theta
$$

the inner surface of the spoiler:

$$
y=y_{0710}(x)+(x-0.55) \tan \theta-0.0012
$$

where $\theta$ is the deployment angle (see Fig. 1a).

The airfoil (1)-(3) is placed in the center of a lens-type computational domain, bounded by two circular arcs,

$$
\begin{aligned}
& \Gamma_{1}: x(y)=105-\left(145^{2}-y^{2}\right)^{1 / 2} \\
& \text { and } \Gamma_{2}: x(y)=-105+\left(145^{2}-y^{2}\right)^{1 / 2},-100 \leq y \leq 100 .
\end{aligned}
$$

The width and height of the domain are 80 and 200, respectively. We set the length $L_{\text {chord }}$ of the airfoil chord to 2.5 $\mathrm{m}$. On the inflow part $\Gamma_{1}$ of the boundary, we prescribe the free-stream Mach number $\mathrm{M}_{\infty}<1$, the angle of attack $\alpha$, and static temperature $T_{\infty}=223.15 \mathrm{~K}$. On the outflow boundary $\Gamma_{2}$, we impose the static pressure $p_{\infty}=26,434 \mathrm{~N} / \mathrm{m}^{2}$. The above values of $T_{\infty}$ and $p_{\infty}$ take place in the standard atmosphere at a height of $10 \mathrm{~km}$. The no-slip condition and vanishing flux of heat are used on the airfoil, which is generally assumed to be smooth. At the end of Section 7, we discuss the lift coefficient calculated for a rough airfoil surface.

The air is treated as a perfect gas, the specific heat at constant pressure of which is $1004.4 \mathrm{~J} /(\mathrm{kg} \mathrm{K})$ and the ratio of specific heats is 1.4 . We adopt the value of $28.96 \mathrm{~kg} / \mathrm{kmol}$ for the molar mass, and use the Sutherland formula for the molecular dynamic viscosity. Initial data are parameters of the uniform free-stream, in which the turbulence level is set to $0.2 \%$.

In Section 8 we examine 3D turbulent flow past a semi wing with airfoil (1)-(3) at spanwise sections and a sweep angle of $15^{\circ}$. The spoiler extends spanwise either from the wing root $z$ $=0$ to wing tip $z=2$ or from $z=0.5$ to $z=1.7$ (Fig. 1b).

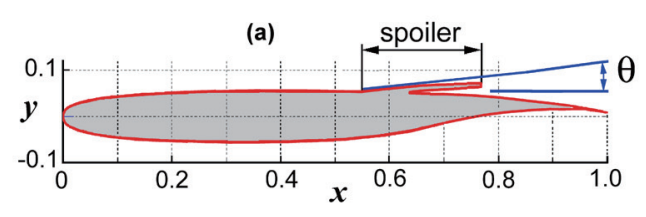

\section{Numerical method}

Solutions of the unsteady Reynolds-averaged NavierStokes equations were obtained with an ANSYS-15 CFX finite-volume solver of second-order accuracy, which is based on a high-resolution scheme by Barth and Jespersen for convective terms [10]. We use an implicit backward Euler scheme for the time-accurate computations. The code uses a linearization of the discretized equations and a multigrid accelerated factorization technique for solving the system of linear equations. Computations of $2 \mathrm{D}$ flow were performed on 3D hybrid meshes with one step $L_{z}=0.01 \mathrm{~m}$ in the $z$-direction. Mesh nodes were clustered in the vicinities of the shock waves, in the wake, and in the boundary layer (see Fig. 2). The non dimensional thickness $y^{+}$of the first mesh layer on the airfoil was less than 1 .

We generally assume the flow is fully turbulent and employ the $k$ - $\omega$ SST turbulence model, which is known to reasonably predict aerodynamic flows with boundary layer separations [11]. At the end of Section 7 we also demonstrate results obtained using a Baseline Reynolds stress turbulence model and a SST Gamma-Theta model of the laminarturbulent transition.

The calculated flow field makes it possible to obtain the normal force $F$ on the airfoil and lift coefficient $C_{\mathrm{L}}=2 F /$ $\left(\rho_{\infty} U_{\infty}^{2} S\right)$, where $U_{\infty}$ is the free-stream velocity module and $S=L_{\text {chord }} \times L_{\mathrm{z}}=0.025 \mathrm{~m}^{2}$ is the wing area, in planform. Test computations of transonic flow and $C_{\mathrm{L}}$ for airfoil (1)-(3) on uniformly refined meshes of 228599, 413428, and 586240 cells showed that solutions obtained on the second and

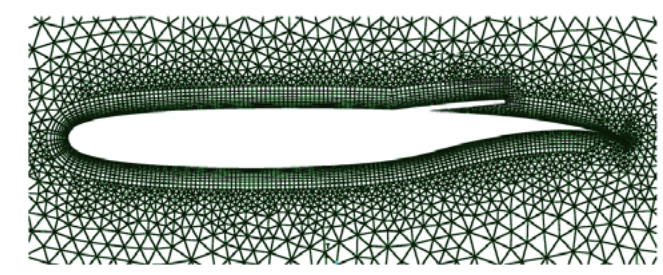

Fig. 2. Sketch of the computational mesh in a vicinity of the airfoil.

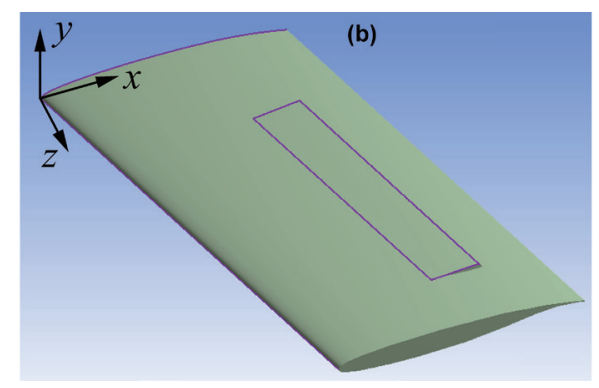

Fig. 1. Sketches of: (a) airfoil (1)-(3) with a spoiler deployment at a positive angle $\theta$, (b) a swept wing with airfoil (1)-(3) at spanwise sections and the spoiler extended over $0.5<z<1.7$; the wing span is 2 airfoil chord lengths; the sweep angle is $15^{\circ}$ deg; the wing is not tapered. 
third meshes almost coincided (see Fig. 3). Global time steps of $2 \times 10^{-5} \mathrm{~s}$ and $10^{-5} \mathrm{~s}$ yielded indistinguishable solutions. That is why we used meshes of about $6 \times 10^{5}$ cells and a step of $2 \times 10^{-5} \mathrm{~s}$ for the study of aerodynamic characteristics of airfoil (1)-(3) at various $\mathrm{M}_{\infty}, \alpha$, and $\theta$. The root-mean-square CFL number (over mesh cells) was about 1.

The solver was verified by computation of a few benchmark transonic flow problems and comparison of solutions with data available in the literature. Fig. 4 shows a good agreement of the calculated lift coefficient $C_{\mathrm{L}}(\alpha)$ for the NASA SC(2)-0712 airfoil at $-4 \leq \alpha^{\circ} \leq 2, \mathrm{M}_{\infty}=0.73$, $\operatorname{Re}=6.5 \times 10^{6}$ with computations performed in [12] using a FLUENT solver and Spalart-Allmaras turbulence model. A slight discrepancy is probably caused by the different turbulence models and different distances from the outer boundary to the airfoil. We also simulated transonic flow past a RAE 2822 airfoil at $-1 \leq \alpha^{\circ} \leq 3$ and obtained the lift coefficient that agreed well with numerical and experimental data documented by several authors (see comparison in [8]).

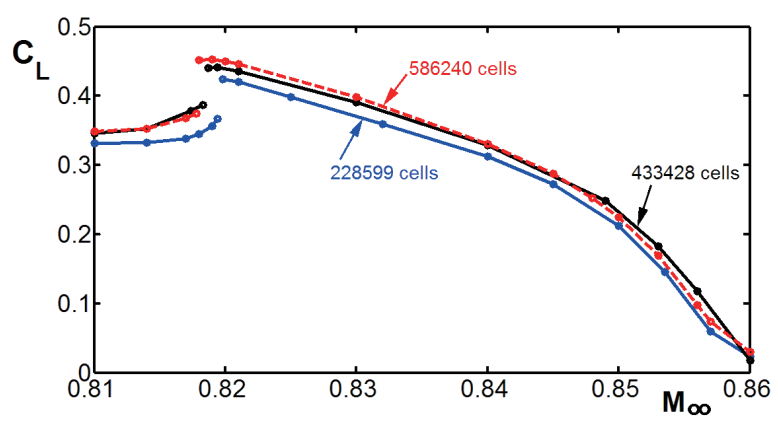

Fig. 3. Lift coefficient for airfoil (1)-(3) versus $M_{\infty}$ calculated on three different meshes using $k-\omega$ SST turbulence model at $\alpha=-0.2^{\circ}$, $\theta=3^{\circ}, \operatorname{Re}=1.4 \times 10^{7}$.

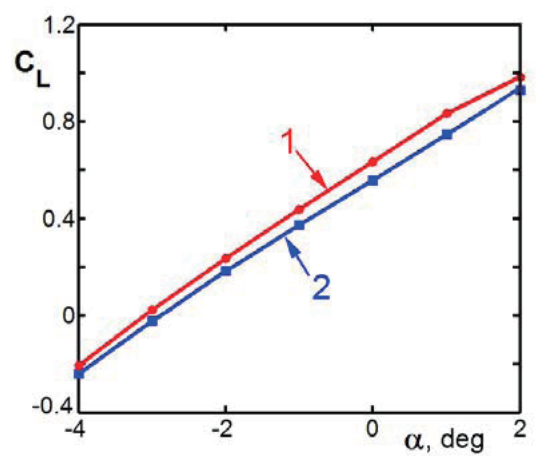

Fig. 4. Lift coefficient versus the angle of attack for a test case of transonic flow over the NASA SC(2)-0712 airfoil at $M_{\infty}=0.73$, $\mathrm{Re}=6.5 \times 10^{6}: 1$ - ANSYS-15 CFX computations, 2 - FLUENT computations by Barrett [12] (reproduced with permission).

\section{Lift coefficient versus $M_{\infty}$ for the airfoil (1) (no spoiler deployment)}

For stationary boundary conditions, computations showed the relaxation to steady solutions (in the sense of mean parameters of turbulent flow) without a buffet onset.

Figure 5 shows the lift coefficient obtained for the steady flow past the NASA SC(02)-0710 airfoil at three angles of attack. As seen, $\mathrm{C}_{\mathrm{L}}$ decreases with the increase of the free-stream Mach number from 0.81 to 0.86 for all $\alpha$ under consideration. This can be explained by a transonic stabilization/freezing effect. Indeed, when $\mathrm{M}_{\infty}$ exceeds 0.81 , the height of the supersonic region on the upper surface of the airfoil becomes large enough, of the order of the airfoil chord length (see Fig. 6a). That is why its growth with increasing $M_{\infty}$ expires due to the stabilization principle [13-15]. As a consequence, the velocity and pressure distributions on the upper surface become almost independent of $\mathrm{M}_{\infty}$. At the same time, beneath the airfoil, the flow velocities are smaller than those above it; thus, the stabilization principle is not valid and the lower supersonic region expands with the increase in $\mathrm{M}_{\infty}$ (see Fig. 6b). This yields a pressure drop on the lower surface and, hence, a drop in the lift coefficient.

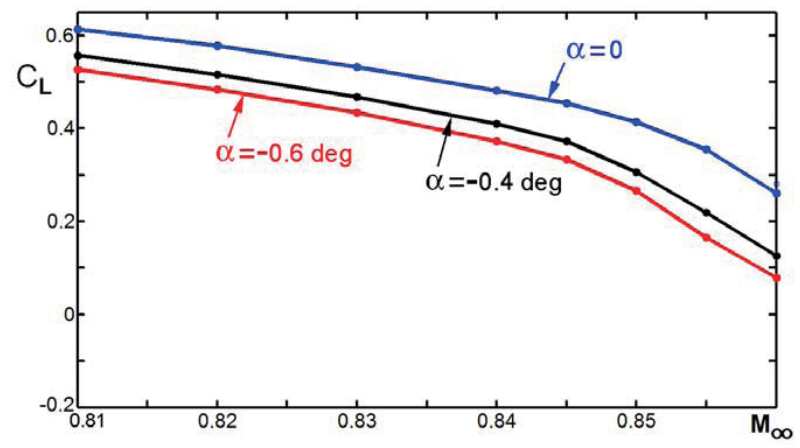

Fig. 5. Lift coefficient $C_{L}$ as a function of $M_{\infty}$ for the NASA SC(02)-0710 airfoil (no spoiler deployment) at three angles of attack.
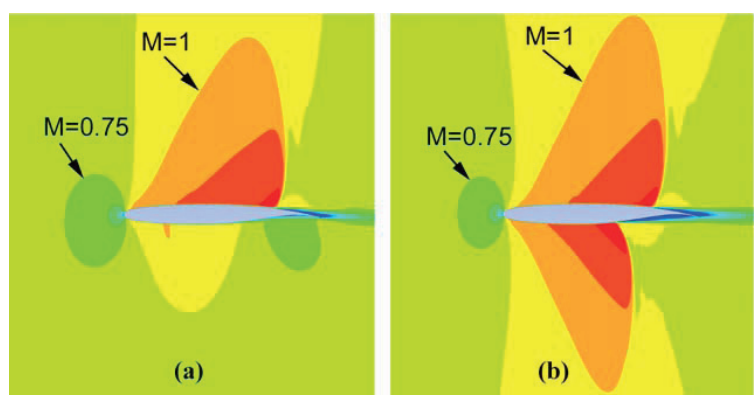

Fig. 6. IsoMachlines in transonic flow past the NASA SC(02)-0710 airfoil (no spoiler deployment) at the angle of attack $\alpha=-0.6^{\circ}$ : (a) $\mathrm{M}_{\infty}=0.82 ;(b) \mathrm{M}_{\infty}=0.86$. 


\section{Lift coefficient behavior for airfoil (1)-(3) at the spoiler deployment angle of $3^{\circ}$}

\subsection{Angles of attack $\alpha=0$ and $\alpha=-0.2^{\circ}$}

Figure 7 shows that plots of the lift coefficient versus $\mathrm{M}_{\infty}$ exhibit a discontinuity, which resides at $\mathrm{M}_{\infty}=0.811$ when $\alpha=0$ and at $\mathrm{M}_{\infty}=0.818$ when $\alpha=-0.2^{\circ}$. The discontinuity is caused by an abrupt coalescence of two supersonic regions on the upper surface of the airfoil with the increase of $\mathrm{M}_{\infty}$. As the Mach number rises further, to 0.860 , the lift coefficient decreases monotonously due to the flow stabilization on the upper surface of the airfoil and an expansion of the supersonic region beneath the airfoil, as in Section 4 .

\section{$5.2 \alpha=-0.4^{\circ}$}

At the smaller angle of attack, $-0.4^{\circ}$, the plot $C_{\mathrm{L}}\left(\mathrm{M}_{\infty}\right)$ exhibits three discontinuities:

- A jump at $\mathrm{M}_{\infty}=0.827$ is caused again by the coalescence of local supersonic regions on the upper surface of the airfoil (Fig. 8a).

- A discontinuous drop of $C_{\mathrm{L}}$ at $\mathrm{M}_{\infty} \approx 0.850$ occurs

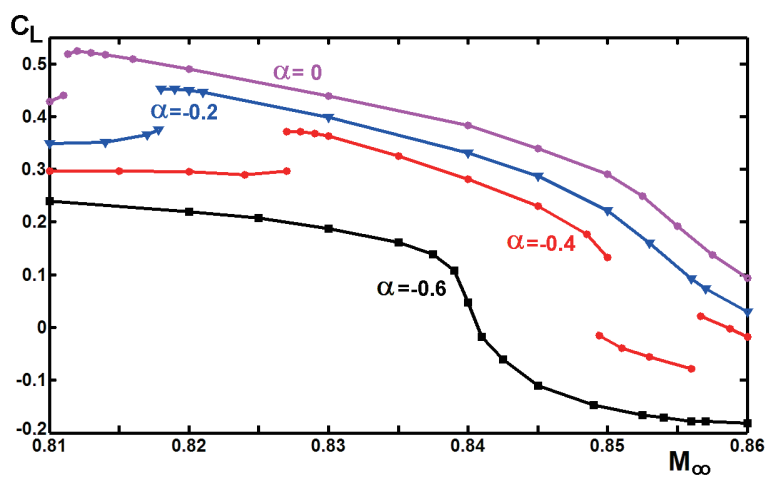

Fig. 7. Lift coefficient as a function of $M_{\infty}$ for airfoil (1)-(3) with the spoiler deflection angle $\theta=3^{\circ}$ at four angles of attack $\alpha$, deg. because of a rupture of the upper supersonic region with increasing $\mathrm{M}_{\infty}$. A closer investigation shows that, as $M_{\infty}$ increases from 0.827 to 0.845 , the supersonic regions expand on both surfaces of the airfoil. The upper supersonic region attains a maximum size at $\mathrm{M}_{\infty}$ $\approx 0.845$ (Fig. 8b), and after that, it turns to a contraction as $\mathrm{M}_{\infty}$ increases further. The contraction is due to the strengthening of the shock wave beneath the airfoil, because the strengthening increases the concavity of streamlines downstream of the shock foot and therefore enhances the slope $\delta$ of the streamlines at the trailing edge (Fig. 9). The rise of $\delta$ influences the flow over the airfoil in the same way as an upward deflection of an aileron does [8]. That is why, with the increase of $\mathbf{M}_{\infty}$, the upper supersonic region shrinks, and it eventually ruptures at $\mathrm{M}_{\infty} \approx 0.850$.

- As $\mathrm{M}_{\infty}$ increases beyond 0.850 , the two supersonic regions on the upper surface turn to an expansion and then a coalescence at $M_{\infty}=0.857$, which produces a third discontinuity in the plot $C_{\mathrm{L}}\left(\mathrm{M}_{\infty}\right)$.

These considerations show that the transonic stabilization principle [15] is not true in case of the spoiler deployment, $\theta$ $=3^{\circ}$, and the angle of attack $\alpha=-0.4^{\circ}$, in contrast to the larger values of $\alpha$.

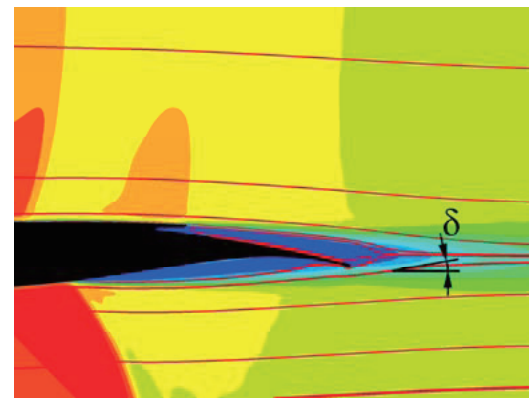

Fig. 9. Streamlines and isoMachlines in a vicinity of the trailing edge at $\mathrm{M}_{\infty}=0.857$, the angle of attack $\alpha=-0.4^{\circ}$, and the spoiler deflection angle $\theta=3^{\circ}$.
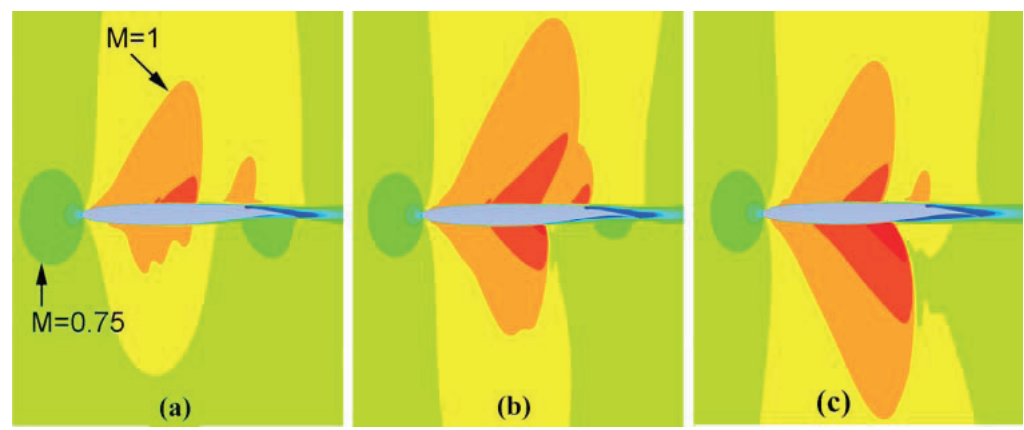

Fig. 8. Evolution of local supersonic regions over airfoil (1)-(3) with the spoiler deflection angle $\theta=3^{\circ}$ at $\alpha=-0.4^{\circ}:$ (a) $M_{\infty}=0.824,\left(\right.$ b) $M_{\infty}=0.846$, (c) $M_{\infty}=0.855$. 
$5.3 \alpha=-0.6^{\circ}$

The lower plot in Fig. 7 is with regard to $\alpha=-0.6^{\circ}$. The considerable steepness of the plot at Mach numbers close to $\mathrm{M}_{\infty} \approx 0.84$ is caused by a rapid expansion of the lower supersonic region with increasing $\mathrm{M}_{\infty}$. An evolution of the double supersonic region on the upper surface of the airfoil is alternate: first, it expands, then shrinks, and after that expands again, as $\mathrm{M}_{\infty}$ increases from 0.81 to 0.86 .

\subsection{Generalization: $C_{L}$ as a function of $M_{\infty}$ and $\alpha$}

Figure 10 presents a surface illustrating the lift coefficient as a function of two parameters, $\alpha$ and $\mathrm{M}_{\infty}$, at $\theta=3^{\circ}$. The surface consists of the lower and upper parts, the projections of which onto the plane $\left(\alpha, \mathrm{M}_{\infty}\right)$ overlap slightly due to a hysteresis in $\alpha$ and $\mathrm{M}_{\infty}$. To determine the edges of the surface, we approached them step by step with sequences $\left(\alpha_{\mathrm{i}}, \mathrm{M}_{\infty, \mathrm{i}}\right)$, and used solutions calculated at a step $i$ as initial data for solving the problem at the step $i+1$.

Figure 11 displays bifurcation curves obtained by projecting the edges of the surface $C_{\mathrm{L}}\left(\alpha, \mathrm{M}_{\infty}\right)$ onto the plane $\left(\alpha, \mathrm{M}_{\infty}\right)$. In the shaded region, projections of the upper and lower parts of the surface $C_{\mathrm{L}}$ overlap. Thus, if given $\alpha^{\prime}, \mathrm{M}_{\infty}^{\prime}$ fall into this region, then there exist two solutions, with single and double supersonic regions on the upper surface

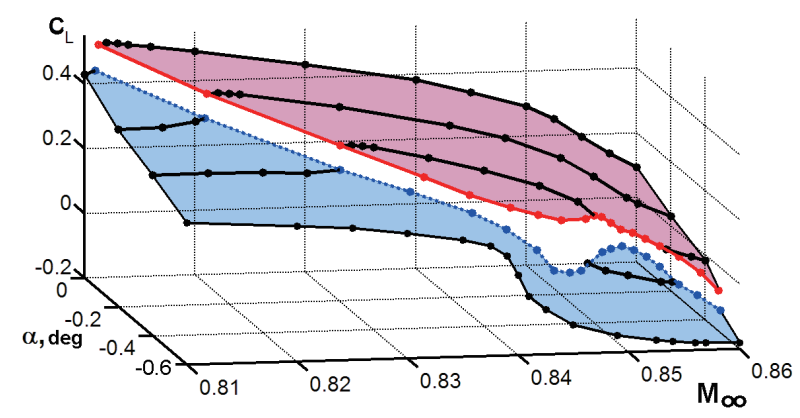

Fig. 10. Lift coefficient $C_{L}$ as a function of $\alpha$ and $M_{\infty}$ for airfoil (1)-(3) in the case of the spoiler deflection angle $\theta=3^{\circ}$.

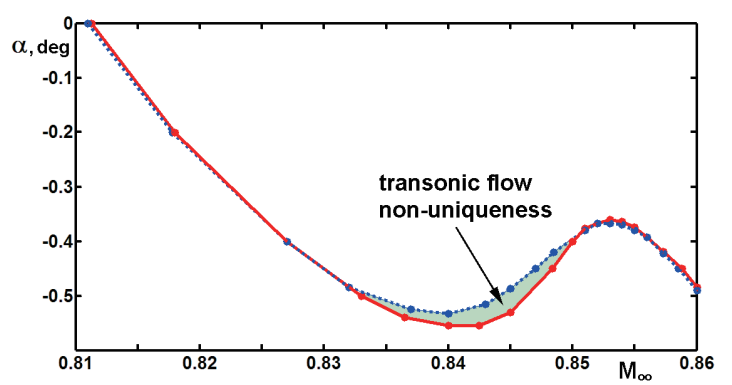

Fig. 11. Bifurcation curves in the plane of the angle of attack $\alpha$ and Mach number $M_{\infty}$ for airfoil (1)-(3) with the spoiler deflection angle $\theta=3^{\circ}$. of the airfoil. The realization of a certain solution in this case depends on the time history of $\alpha^{\prime}$ and $\mathrm{M}_{\infty}^{\prime}$ (i.e., on the way in which $\alpha(t) \rightarrow \alpha^{\prime}$ and $\left.\mathrm{M}_{\infty}(t) \rightarrow \mathrm{M}_{\infty}^{\prime}\right)$.

We note that the physical time required for the flow relaxation to a steady state at given $\alpha^{\prime}$ and $\mathrm{M}_{\infty}^{\prime}$ is about $2 \mathrm{~s}$ if the point $\left(\alpha^{\prime}, \mathrm{M}_{\infty}^{\prime}\right)$ is far enough from the bifurcation curves shown in Fig. 11. The relaxation time rises to about $7 \mathrm{~s}$ when the point is located close to the bifurcation curves.

\section{Aerodynamic coefficient behavior at a spoiler deployment angle of $6^{\circ}$}

If the spoiler deflection angle is increased to $6^{\circ}$, then beneath the airfoil the flow velocity rises and static pressure decreases. As a consequence, the lift coefficient decreases for all $\alpha$ and $\mathrm{M}_{\infty}$ under consideration. Fig. 12 presents plots of the lift coefficient versus $\mathrm{M}_{\infty}$ at $\alpha=0,0.6^{\circ}$, and $-0.4^{\circ}$. A plot with regard to $\alpha=-0.6^{\circ}$ is omitted from the figure, as it drops to values of little practical interest. It can be seen that the Mach numbers $0.835<\mathrm{M}_{\infty}<0.850$ are most adverse from the viewpoint of aerodynamic loads on the airfoil, as they allow considerable jumps of $C_{\mathrm{L}}$ when $\alpha$ varies between 0 and $0.6^{\circ}$.

In Fig. 12, the monotonous decrease of $C_{\mathrm{L}}$ with the increase of $\mathrm{M}_{\infty}$ is caused by an expansion of the supersonic region beneath the airfoil, even though the flow does not stabilize on the upper surface, in contrast to the flow behavior discussed

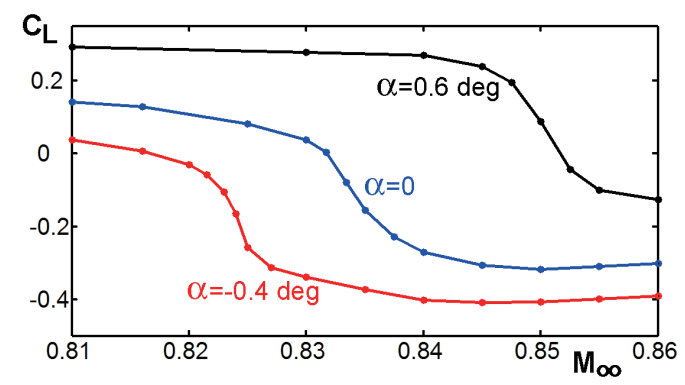

Fig. 12. Lift coefficient $C_{L}$ versus $M_{\infty}$ for airfoil (1)-(3) in the case $\theta=6^{\circ}$.

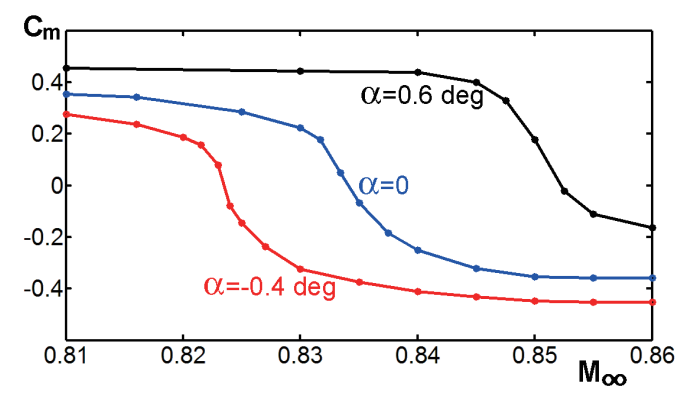

Fig. 13. Pitching moment coefficient $C_{m}$ versus $M_{\infty}$ for airfoil (1)-(3) in the case $\theta=6^{\circ}$. 
in Sections 4 and 5.1. Indeed, when $\alpha=0$ and $\alpha=-0.4^{\circ}$, the supersonic region on the upper surface of the airfoil is either small or disappearing and the flow velocity is much smaller than those at which the transonic stabilization principle is true. When $\alpha=0.6^{\circ}$, there is a noticeable supersonic region on the upper surface. However, computations show its considerable evolution with increasing $\mathrm{M}_{\infty}$; thus, the stabilization principle is also not true.

Figure 13 demonstrates the calculated pitching moment coefficient $C_{\mathrm{m}}=2 \mathrm{M}_{\mathrm{z}} /\left(\rho_{\infty} U_{\infty}{ }^{2} S \times 1[\mathrm{~m}]\right)$ versus $\mathrm{M}_{\infty}$ for three angles of attack, where $\mathrm{M}_{\mathrm{z}}$ is calculated about the reference point $x=y=0$. As seen, the behavior of $C_{\mathrm{m}}$ is similar to that of $C_{\mathrm{L}}$.

\section{Miscellania}

The effect of spoiler deployment on the lift coefficient is also illustrated in Fig. 14, which collects plots $C_{\mathrm{L}}\left(\mathrm{M}_{\infty}\right)$ for three values of $\theta$ at the angle of attack $\alpha=-0.4^{\circ}$. As seen, the lift coefficient changes drastically if $0.825<\mathrm{M}_{\infty}<0.850$ and $\theta$ switches between $3^{\circ}$ and $6^{\circ}$. For example, when $M_{\infty}=0.83$, the lift coefficient jumps from -0.34 at $\theta=6^{\circ}$ to 0.36 at $\theta=3^{\circ}$. When $\mathrm{M}_{\infty}=0.81$, the jump is smaller, from 0.04 to 0.30 .

The drastic changes of $C_{\mathrm{L}}$ at the Mach number $\mathrm{M}_{\infty}=0.83$ were confirmed by computations of the lift coefficient as a function of time for impulse changes of the spoiler deployment angle $\theta$ that switches from $3^{\circ}$ to $6^{\circ}$ and vice versa every $0.6 \mathrm{~s}$ (see Fig. 15a). The amplitude of lift coefficient oscillations does not attain the steady-state extrema, -0.34 and 0.36 , because the time $\Delta t=0.6 \mathrm{~s}$ is insufficient for the flow relaxation after a switch of $\theta$. Fig. $15 \mathrm{~b}$ shows that a decrease of the free-stream Mach number from 0.83 to 0.81 almost halves the amplitude of lift coefficient oscillations. The flow relaxation is slower in Fig. 15a than in Fig. 15b in agreement with the note at the end of Section 5.4, because the data $\mathrm{M}_{\infty}=0.83, \alpha=-0.4^{\circ}$ are close to the bifurcation region, whereas $M_{\infty}=0.81, \alpha=-0.4^{\circ}$ are distant from it. If the

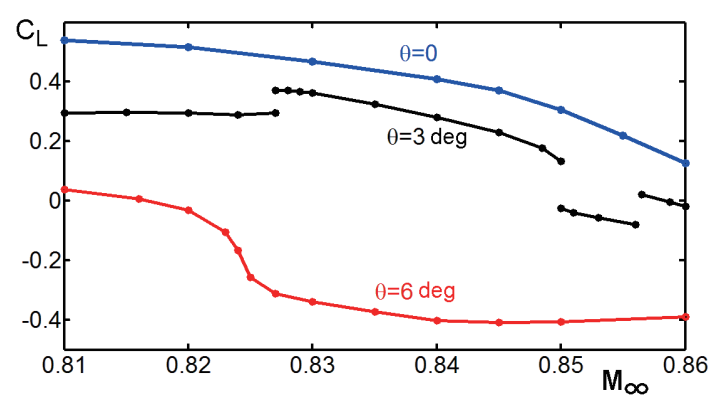

Fig. 14. Lift coefficient $C_{L}$ versus $M_{\infty}$ for three spoiler deflection angles $\theta$ at the angle of attack of $-0.4^{\circ}$ period of spoiler oscillations is increased (e.g., if $\Delta t$ is tripled) then extrema of the lift coefficient oscillations rise at $\mathrm{M}_{\infty}=$ 0.83 and become closer to the steady-state values; thus, the difference between the amplitudes of $C_{\mathrm{L}}(t)$ at $\mathrm{M}_{\infty}=0.81$ and $\mathrm{M}_{\infty}=0.83$ rises.

Figure 16 displays the lift coefficient calculated using different turbulence models. For the smooth airfoil surface, the figure shows good agreement with the results obtained using the $k$ - $\omega$ SST turbulence model and BSL Reynolds Stress model, which is based on differential transport equations with a low-Reynolds formulation near the solid walls [16]. Also, there is good agreement of the plots obtained using the $k$ - $\omega$ SST model for the smooth surface and a rough one with a sand-grain roughness of $10^{-5} \mathrm{~m}$. The SST GammaTheta model of laminar-turbulent transition, which solves transport equations for intermittency and momentum thickness Reynolds number [17], predicts a shift of the lift coefficient jump to a smaller value of the Mach number, $M_{\infty}$ $=0.814$. This is caused by a faster development of the double
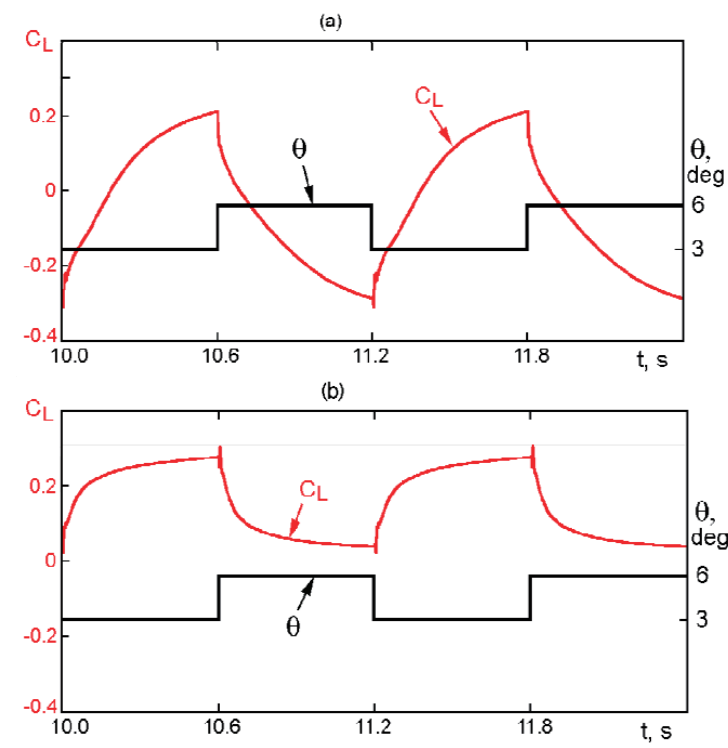

Fig. 15. Lift coefficient $C_{\mathrm{L}}$ and spoiler deployment angle $\theta$ as functions of time $t$ at $\alpha=-0.4^{\circ}$ : (a) $\mathrm{M}_{\infty}=0.83$, (b) $\mathrm{M}_{\infty}=0.81$.

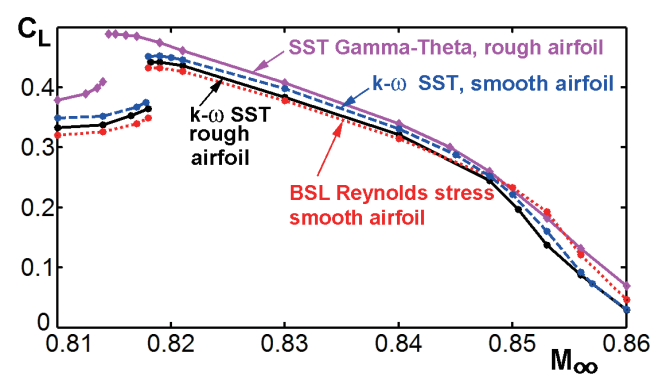

Fig. 16. Lift coefficient for airfoil (1)-(3) calculated using different turbulence models at $\alpha=-0.2^{\circ}, \theta=3^{\circ}$. 
supersonic region on the airfoil with increasing $\mathrm{M}_{\infty}$.

\section{3D wings}

To examine the influence of 3D phenomena on the configuration of local supersonic regions, we performed simulations of transonic flow past the wing depicted in Fig. $1 \mathrm{~b}$ at a spoiler deflection angle of $3^{\circ}$. The wing is inclined at an angle of attack of $-0.4^{\circ}$ and placed in the computational domain $10<x, y<10,0<z<12$. A hybrid mesh is constituted by $7.7 \mathrm{mln}$ prisms in 32 layers on the wing and $24.3 \times 10^{6}$ tetrahedrons in the remaining region. We prescribe the no-slip condition on the wing, a condition of symmetry on the wing root plane $z=0$, and the free-slip condition on the bottom, top, and side boundaries $y= \pm 10, z=12$. Conditions on the inflow and outflow boundaries are the same as in the $2 \mathrm{D}$ case.

The calculated flow field exhibits a curious shape of the local supersonic region on the upper surface of the wing at $\mathrm{M}_{\infty}=0.84, \alpha=-0.4^{\circ}$ (see Fig. 17a). The supersonic region ruptures over the spoiler in agreement with the results obtained for 2D flow in Section 5.2. Fig. 17b demonstrates isoMachlines at three spanwise sections.

Figure 18a displays the sonic surface in the case when $\mathrm{M}_{\infty}$ $=0.84$ and the spoiler (2),(3) is extended over the full span $0 \leq z \leq 2$. The extension influences the supersonic region considerably, so that it splits into two parts. As $\mathrm{M}_{\infty}$ increases, from 0.84 to 0.877 , the parts approach each other (see Fig. $18 \mathrm{~b}$ ), and then they coalesce at $\mathrm{M}_{\infty} \approx 0.885$. The latter Mach number is larger than the value of 0.828 , which triggers the coalescence of supersonic regions and a jump of $C_{\mathrm{L}}$ in 2D flow (see the plot respective to $\alpha=-0.4^{\circ}$ in Fig. 7). This is explained by the high sensitivity of the flow field to the nonzero spanwise velocity component in the case of a swept wing.

To verify the 3D modeling, we performed simulations of transonic flow for a commonly used test case of the flow past an ONERA M6 wing at $\mathrm{M}_{\infty}=0.84, \alpha=3.06^{\circ}$. The Reynolds number based on the mean chord was 11.72 million. Fig.19 shows a comparison of the surface pressure coefficients as computed with the CFX solver and a Cart3D-IBL one, the
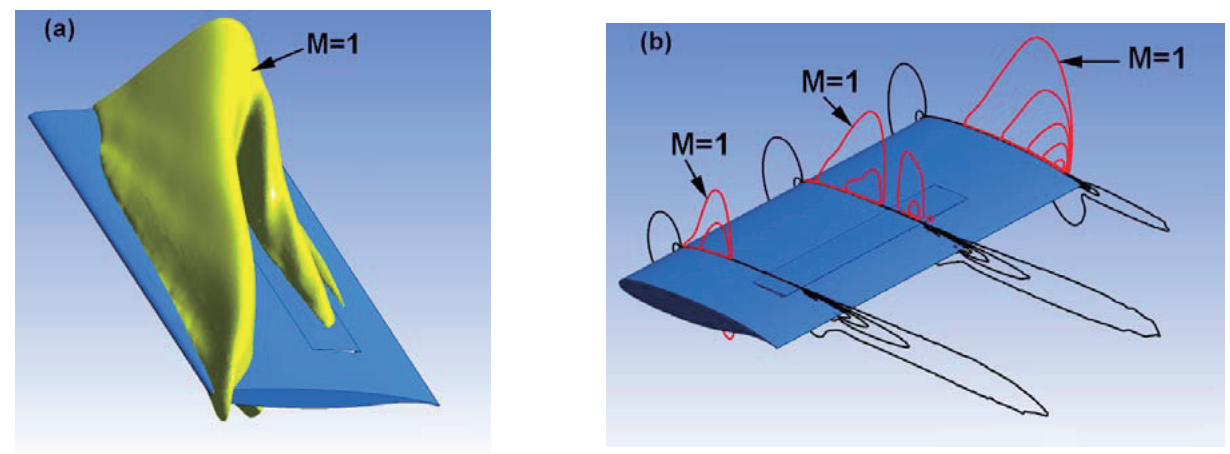

Fig. 17. Transonic flow over the wing sketched in Fig. $1 \mathrm{~b}$ with the sweep angle of $15^{\circ}$. The spoiler (2),(3) extends over $0.5<z<1.7$, the spoiler deflection angle $\theta=3^{\circ}$, the angle of attack $\alpha=-0.4^{\circ}$, free-stream Mach number $M_{\infty}=0.84$; (a) sonic surface on the wing, (b) isoMachlines at three spanwise sections $z=0, z=0.8, z=1.6$.
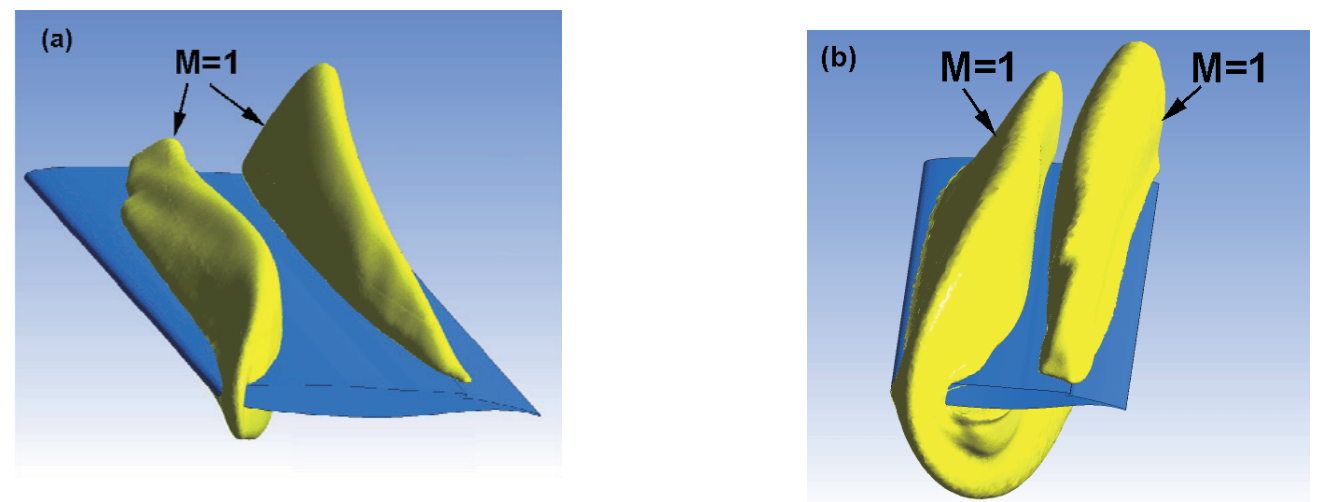

Fig. 18. Sonic surface on the wing sketched in Fig. $1 \mathrm{~b}$ with the sweep angle of $15^{\circ}$. The spoiler (2),(3) extends over the full span $0<z<2$, the spoiler deflection angle $\theta=3^{\circ}$, the angle of attack $\alpha=-0.4^{\circ}$ : (a) $M_{\infty}=0.84$, (b) $M_{\infty}=0.877$. 

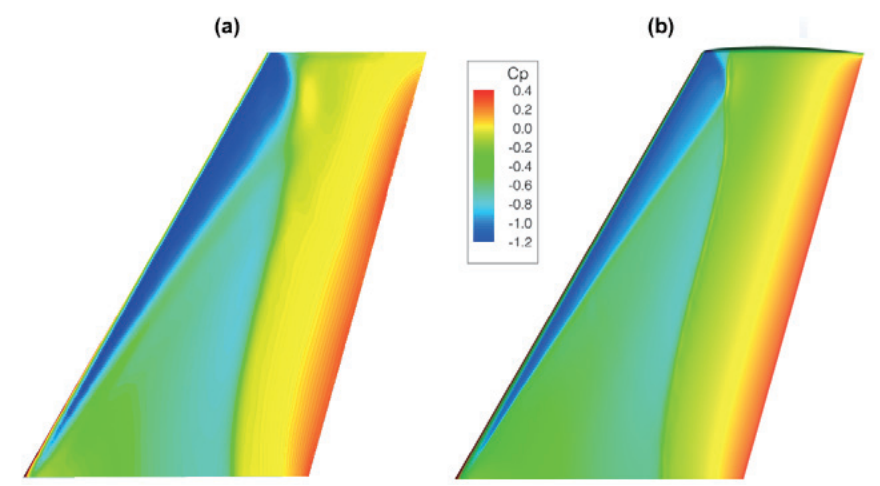

Fig. 19. A comparison of the pressure coefficient $C_{p}$ contours on the upper surface of ONERA M6 wing at $\alpha=3.06^{\circ}, M_{\infty}=0.84$ : (a) computations with the CFX solver, (b) computations with a Cart3D-IBL by Rodriguez et al. [18] (reproduced with permission).

results of which are in good agreement with experimental data [18]. The contours are very similar, though the CFX solution exhibits a bit weaker shock near the wing tip where two shock waves merge into one.

\section{Conclusions}

The numerical simulations have revealed that, in the band $0.83 \leq \mathrm{M}_{\infty} \leq 0.85$, transonic flow over airfoil (1)(3) is extremely sensitive to perturbations of free-stream parameters and spoiler deployments. In this band, if the spoiler is deflected at an angle of $3^{\circ}$, then slight variations of the angle of attack $\alpha$ between $-0.6^{\circ}$ and $-0.2^{\circ}$ produce crucial changes in the lift and pitching moment coefficients. Also at a spoiler deflection angle of $6^{\circ}$, fluctuations of $\alpha$ between 0 and $0.6^{\circ}$ produce drastic jumps of the lift coefficient between -0.3 and 0.4 . The fluctuations of the angle of attack may occur in practice at vertical gusts of wind in the cruise flight of civil or transport aircraft.

Moreover if free-stream parameters are stationary but their values are unfavorable, (e.g., $\alpha=-0.4^{\circ}$ ), then variations in the spoiler deployment from 3 to $6^{\circ}$, and vice versa, result in dramatic aerodynamic loads on the airfoil.

Computations of 3D flow over a swept wing showed that adverse values of the Mach number $\mathrm{M}_{\infty}$ are larger than those in 2D flow. Further work is needed to advance the prediction of unfavorable flight conditions and to contribute to flight control algorithms.

\section{Acknowledgements}

This research was performed using computational resources provided by the Computational Center of St. Petersburg State University (http://cc.spbu.ru). The work was supported by the Russian Foundation for Basic Research under grant no. 13-08-00288.

\section{References}

[1] Stuckey, R. A., "A comparison of spoiler aerodynamic characteristics as estimated from flight", IAC-97 International Aerospace Congress Proceedings, Vol. 2. Sydney, NSW, 1997, pp. 721-731.

[2] Fillola, G., Le Pape, M.-C. and Montagnac, M., "Numerical simulations around wing control surfaces", ICAS 2004 Proceedings, Yokohama, 2004.city name, year. http:// www.cerfacs.fr/ cfdbib/repository/TR_CFD_04_87.pdf, 2004.

[3] Méheut, M., Atinault, O. and Hantrais-Gervois, J.L., "elsA and TAU assessment for wing control surfaces", Research Report, TP 2011-102, ONERA, Toulouse, France, 2011.

[4] Dimitrov, D., "Unsteady aerodynamics of wings with an oscillating flap in transonic flow", 8th PEGASUS-AIAA Student Conference, Poitiers, Frankreich, 2012.

[5] Reimer, L. and Heinrich, R., "Modeling of movable control surfaces and atmospheric effects", Notes on numerical fluid mechanics and multidisciplinary design, Vol. 123, 2013, pp. 183-206.

[6] Blanc, F., Roux, F.-X. and Jouhaud, J.-Ch., "Numerical methods for control surfaces aerodynamics with flexibility effects". International Forum on Aeroelasticity and Structural Dynamics 2009, CERFACS, Toulouse, France, 2009, pp. 1-15,

http://www.cerfacs.fr/ cfdbib/repository/TR_ CFD_09_54.pdf

[7] Kuzmin, A., "Non-unique transonic flows over airfoils", Computers and Fluids, Vol. 63, 2012, pp. 1-8.

[8] Kuzmin, A., "Transonic flow past a Whitcomb airfoil with a deflected aileron", Internat. J. of Aeronautical and 
Space Sciences, Vol. 14, No. 3, 2013, pp. 210--214.

[9] Harris, Ch.D., NASA supercritical airfoils. A matrix of family-related airfoils, NASA Technical Paper, No. 2969, Langley Research Center, 1990.

[10] Barth, T.J. and Jespersen, D.C., "The design and application of upwind schemes on unstructured meshes", AIAA Paper, 89-0366, 1989, pp. 1-12.

[11] Menter, F.R., "Review of the Shear-Stress Transport turbulence model experience from an industrial perspective", Internat. J. Comput. Fluid Dynamics, Vol. 23, 2009, pp. 305316.

[12] Barrett, Th.R., Aerodynamic design optimization using flow feature parametrization, $\mathrm{PhD}$ thesis, University of Southampton, School of Engineering Sciences, Southampton, UK, 2007.

[13] Christianovich, S.A., Galperin, V.G., Gorsky, I.P. and
Kovalev, A.P., The physical basis for nearsonic aerodynamics, TsaGI, Moscow (in Russian), 1948.

[14] Zierep, J., "The freezing property (stabilization law) of transonic flows", Acta Mechanica, Vol. 108, 1995, pp. 219-223.

[15] Cook, L.P. and Zeigler, F.J., "The stabilization law for transonic flow". SIAM J. Appl. Math., Vol. 46, No. 1, 1986, pp. $27-48$.

[16] Sharcnet, "Computing tomorrow's solutions", https:// www.sharcnet.ca/Software/Fluent14/help/cfx_thry/ i1303039.html , 2013.

[17] Langtry, R.B. and Menter, F.R., “Transition modeling for general CFD applications in aeronautics", AIAA Paper, No. 2005-522, 2005, pp.1-14.

[18] Rodriguez, D.L., Sturdza, P., Suzuki, Y. and Martinsrivas, $\mathrm{H}$., "A rapid, robust, and accurate coupled boundarylayer method for Cart3D”, AIAA Paper, No. 2012-0302, 2012. 